\title{
Sistema de Detecção de Intrusão em Redes de Computadores Utilizando Máquinas de Vetores de Suporte
}

\author{
Heitor Scalco Neto* Wilian Soares Lacerda ${ }^{* *}$ \\ * Núcleo de Informática - Universidade Federal de Santa Maria \\ Palmeira das Missões - RS - CEP 98300-000 - Brasil \\ (e-mail: heitorscalco@hotmail.com) \\ ** Departamento de Automática - Universidade Federal de Lavras \\ Lavras - MG - CEP 37200-000 - Brasil (e-mail: lacerda@ufla.br)
}

\begin{abstract}
This paper presents a Network Intrusion Detection System $(N I D S)$ with real-world traffic using Support Vector Machine $(S V M)$. Two databases were used for the experiments: the network traffic database provided by the $I S C X$ (Information Security Center of Excellence); and a test database created during of the experiments. Results obtained with the use of the Support Vector Machines technique show correct rates of $95 \%$, thus confirming the feasibility of using the $S V M$ technique to solve problems of classifying traffic in computer networks in real environment.

Resumo: Este artigo apresenta um Sistema de Detecção de Intrusão em Redes de Computadores (NIDS - Network Intrusion Detection System), com tráfego de ambiente real, utilizando Máquina de Vetores de Suporte ( $S V M$ - Support Vector Machine). Para os experimentos, foram utilizadas duas bases de dados: a base de dados de tráfego de rede disponibilizada pela ISCX (Information Security Centre of Excellence); e uma base de dados de testes, criada no decorrer dos experimentos. Resultados obtidos com a utilização da técnica de Máquinas de Vetores de Suporte mostram taxas de acertos em torno de 95\%, afirmando assim a viabilidade da utilização da técnica de SVM para resolver problemas de classificação de tráfego de redes de computadores, em ambiente real.
\end{abstract}

Keywords:

Pattern recognition; vector machine support; network intrusion; traffic detection.

Palavras-chaves: Reconhecimento de padrões; máquina de vetor de suporte; intrusão em redes; detecção de tráfego.

\section{INTRODUÇÃO}

Em virtude do crescimento exponencial do número de ataques cibernéticos (CERT.BR, 2019) nos últimos anos, uma tecnologia tornou-se aliada aos administradores de rede: os Sistemas de Detecção de Intrusão em Redes de Computadores (NIDS - Network Intrusion Detection Systems). Essa ferramenta tem importância fundamental para garantir a confiabilidade e disponibilidade em uma rede de computadores. Dessa forma, este artigo propõe uma metodologia para o desenvolvimento de um NIDS por anomalias, Open-Source, e utilizando Máquinas de Vetores de Suporte (SVM - Support Vector Machines).

A técnica de $S V M$ é aplicada e avaliada, com intuito de estabelecer a eficácia do método para a detecção de intrusão em redes de computadores. Para que o NIDS fosse capaz de operar em ambiente real, foi necessário o desenvolvimento de uma API (Application Programming Interface) que tem como objetivo capturar o tráfego de rede e realizar o préprocessamento da informação. Sendo assim, foi possível realizar experimentos com diferentes infraestruturas de rede e em ambiente real. O treinamento dessa técnica foi realizado com a base de dados de tráfego de rede ISCX (Information Security Centre of Excellence) ${ }^{1}$, a qual é composta por tipos de tráfego variados (Ex.: VOIP, SSH, HTTP, HTTPS, FTP, entre outros).

Por meio da $A P I$ desenvolvida, criou-se uma base de dados auxiliar para testes com uma infraestrutura de rede reduzida em relação a apresentada pela ISCX e com diferentes sistemas operacionais e aplicações. Essa base de dados permite que testes de eficiência do método sejam realizados em diferentes infraestruturas e com diversos modos de utilização. A API permite seu uso de três diferentes formas: (i) Online, em que funciona da mesma forma que um NIDS; (ii) Captura de Dados, em que é utilizada para criar e pré-processar bases de dados; (iii) Leitura de Bases de Dados, em que permite a extração de características de bases de dados já existentes.

Este artigo tem como principais contribuições os seguintes tópicos: (i) avaliação da técnica de Máquinas de Vetores de Suporte para o problema de detecção de intrusão em redes de computadores; (ii) desenvolvimento de uma API para

\footnotetext{
$\overline{1 \text { http://www.unb.ca/research/iscx/ }}$
} 
captura de pacotes, pré-processamento e integração com diferentes técnicas de Inteligência Computacional; (iii) utilização de características independentes de softwares e/ou hosts em NIDS.

\section{REVISÃO BIBLIOGRÁFICA}

Esta seção apresenta uma breve revisão bibliográfica sobre Sistemas de Detecção de Intrusão e Máquinas de Vetores de Suporte.

\subsection{Sistemas de Deteç̧ão de Intrusão}

O termo Detecção de Intrusão define-se como o processo de monitorar eventos que estão ocorrendo em um sistema computacional ou em uma rede de computadores, buscando tráfego intrusivo. Incidentes de segurança possuem várias causas como: a propagação de um malware, atacantes tentando elevar privilégios para acessar sistemas não autorizados, negação de serviço, entre outros (Karen Scarfone, 2007).

Um Sistema de Detecção de Intrusão em Redes (ou NIDS) trabalha analisando exclusivamente o tráfego de rede sem utilizar informações específicas de hosts (Ex.: utilização de memória, processamento, interfaces), geralmente utilizando uma interface em modo promíscuo e funcionando como um sniffer ${ }^{2}$ (Uchoa, 2009). Esse tipo de sistema geralmente atua com um ou mais sensores na rede e uma estação de monitoramento. Quando um sensor detecta uma atividade anormal na rede, um alerta é transmitido para a estação de monitoramento que informará ao administrador da rede sobre a situação. A Figura 1 apresenta um exemplo de posicionamento de um NIDS na rede (Scalco Neto et al., 2015).

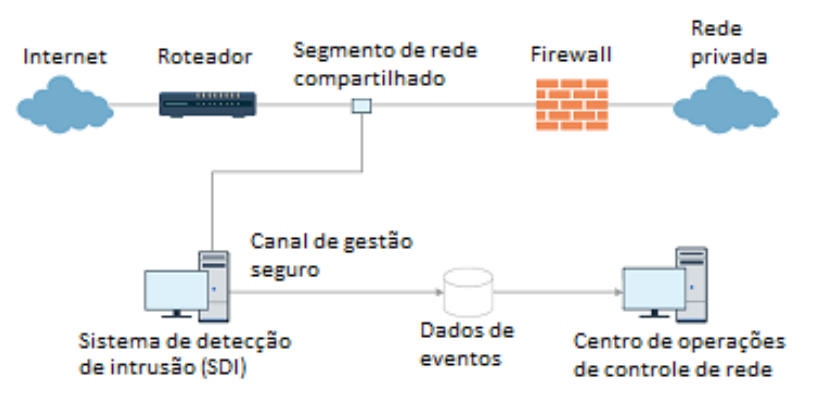

Figura 1. Posicionamento de um NIDS na rede (Scalco Neto et al., 2015)

A forma na qual os NIDS reconhecem uma ação intrusiva pode ser baseada em assinaturas ou baseado em anomalias. Sistemas de detecção baseados em assinaturas identificam ataques através da análise de assinaturas previamente configuradas sobre o comportamento padrão de algum tipo de ataque. No caso do NIDS baseado em anomalias, é analisado um padrão da rede, ou host, classificando o tráfego em anômalo ou normal. A principal vantagem de um NIDS baseado em anomalia é que é possível detectar ataques desconhecidos, o que não acontece na detecção por assinatura (Wang, 2009).

\footnotetext{
2 Ferramenta de captura de tráfego de rede
}

Um método para o reconhecimento de intrusão em redes de computadores pode ser a utilização de técnicas de Inteligência Computacional como Máquinas de Vetores de Suporte. A técnica de Máquinas de Vetores de Suporte é descrita na próxima seção.

\subsection{Máquinas de Vetores de Suporte}

Máquinas de Vetores de Suporte (SVM - Support Vector Machines) é uma técnica utilizada para o treinamento de classificadores baseada no conceito da minimização do risco estrutural. Essa técnica destaca-se por três características principais: possuem uma fundamentação teórica plausível; podem alcançar alto desempenho em certas aplicações (Santos, 2002); e possuem um grande poder de generalização (Rodrigues et al., 2007).

A técnica de $S V M$ consiste em utilizar classificadores lineares que tem como objetivo separar os dados em duas ou mais classes por meio da criação de um hiperplano de separação. Um hiperplano ótimo separa os dados de forma que a margem seja a maior possível, sendo que a mesma é definida através da soma das distâncias entre os pontos positivos e negativos mais próximos do hiperplano, o qual é criado por meio de um conjunto finito de dados de treinamento. Esses pontos compõem os "vetores de suporte" demarcados na Figura 2 (Rodrigues et al., 2007). Existem diversos tipos de $S V M$, cada qual com uma finalidade específica. O tipo de $S V M$ utilizado neste trabalho foi o SVC (Support Vector Classification), o qual realiza o processo de classificação em duas ou mais classes.

Figura 2. Classificação de um conjunto de dados utilizando SVM linear (Rodrigues et al., 2007).

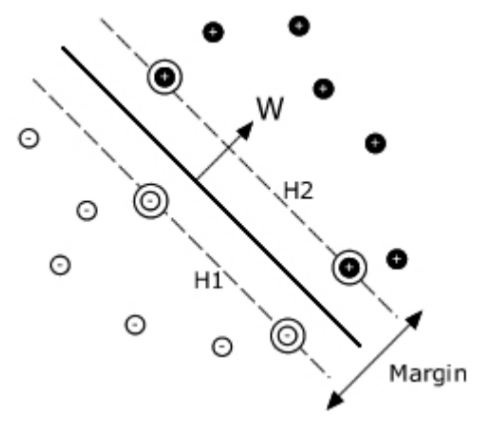

Tipos de Kernel De acordo com Ben-Hur and Weston (2010), um kernel é um algoritmo que depende do produto escalar de um determinado conjunto de dados. Em outras palavras, um kernel é uma função de similaridade que é utilizada, dependendo do conjunto de dados, juntamente com o algoritmo de aprendizado.

Em muitos casos, classificadores (kernels) não lineares proporcionam melhores taxas de acertos. Porém, conforme apresentado em Hsu et al. (2003), em alguns casos um classificador linear pode se sobressair, são eles:

- Número de amostras $<<$ Número de Características.

- Número de amostras e Número de Características muito grandes.

- Número de amostras >> Número de Características. 
Os tipos de kernel mais comumente utilizados, de acordo com Hsu et al. (2003), são: linear, polinomial, RBF e sigmoide. É recomendado fortemente que o treinamento inicial do $S V M$ seja realizado com kernel linear, o qual pode trazer algumas vantagens tais como a economia de tempo e simplicidade do modelo. Caso o aprendizado utilizando kernel linear não resulte em um classificador eficaz, aconselha-se a tentativa com outros modelos de kernel.

Parâmetros de Treinamento O hiperplano criado no SVM pode ser "moldado" por meio dos seguintes parâmetros: gamma e custo. O parâmetro custo (Figura 3) é utilizado para atribuir valores de penalidade para erros no treinamento. Um valor de custo alto faz com que os dois pontos próximos ao hiperplano afetem sua orientação, resultando em um hiperplano de separação que é muito próximo ao de outras classes de dados. Por outro lado, um valor de custo baixo faz com que a orientação do hiperplano não seja tão afetada por erros de treinamento. Sendo assim, é possível conseguir uma margem maior de separação. A Figura 3 apresenta o resultado da utilização de diferentes valores de custo em um determinado processo de classificação.

Figura 3. Resultado de diferentes valores de custo na classificação (Ben-Hur and Weston, 2010).
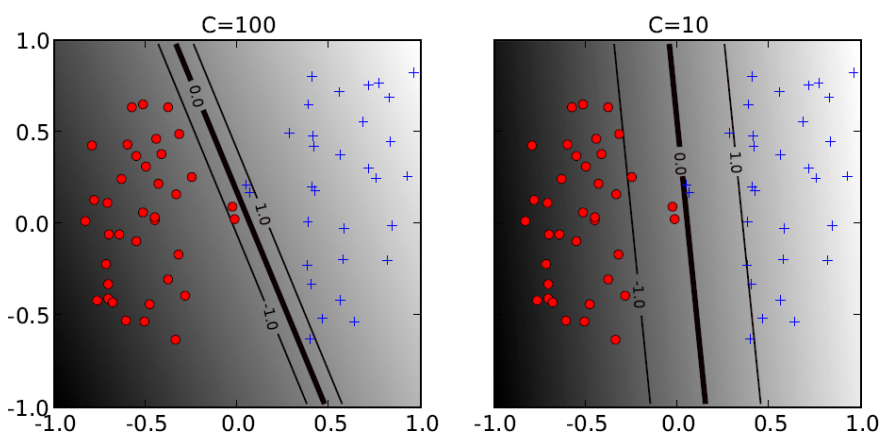

Já o parâmetro gamma define a "flexibilidade" da fronteira de decisão (Ben-Hur and Weston, 2010). À medida que o gamma aumenta, a expansão da localidade do vetor de suporte também aumenta, com tendência para uma maior curvatura da fronteira de decisão (dependendo do conjunto de dados). A Figura 4 representa a utilização de variados valores de gamma. Dessa forma, os parâmetros custo e gamma precisam formar uma combinação que permita ao SVM realizar a classificação de forma eficaz. Cabe ressaltar que o parâmetro gamma só é empregado com a utilização de kernels não lineares (Shalev-Shwartz et al., 2011).

\section{MATERIAIS E MÉTODOS}

Realizar a análise e classificação de tráfego de rede em ambiente real é sempre um desafio. De acordo com Scalco Neto et al. (2015), a dificuldade em construir um NIDS eficiente é fazer com que o número de verdadeiros positivos seja grande, e em contra partida, o número de falsos positivos deve ser pequeno ou zero. Além deste desafio, a grande quantidade de informações que pode-se extrair de uma conexão de rede pode dificultar a detecção
Figura 4. Diferentes Valores de gamma para função Gaussiana (Ben-Hur and Weston, 2010).
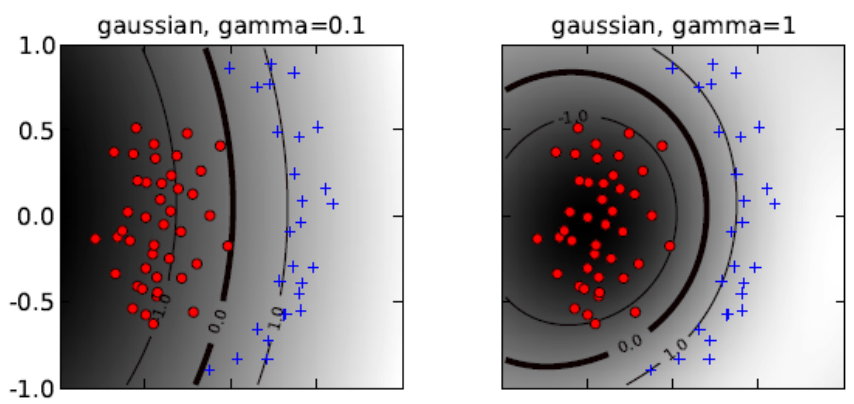

pois, em grande parte das vezes, uma intrusão pode ser caracterizada por uma ou mais variações das características de uma conexão.

Com intuito de buscar a solução para este problema, foi utilizada a técnica de $S V M$ e um conjunto de características extraídas de uma conexão. A técnica de $S V M$ apresenta um grau avançado de generalização, podendo beneficiar os resultados da classificação de tráfego intrusivo em redes de computadores. Para os experimentos com SVM, foi utilizada a plataforma Microsoft Machine Learning Studio (Microsoft, 2019). O processo de resolução do problema proposto é apresentado em detalhes no decorrer desta seção.

\subsection{A Base de Dados ISCX 2012}

Durante as últimas décadas, detecção de intrusão por anomalias atraiu a atenção de muitos pesquisadores. A base de dados KDD Cup'99 (Stolfo et al., 2000) é atualmente uma das bases de dados mais utilizadas para avaliação e treinamento de sistemas detectores de intrusão (Salem et al., 2014). Porém, de acordo com Uchoa (2009), ela não reflete mais a infraestrutura, serviços e tráfego de uma rede de computadores atual. Desta forma, foi necessária a utilização de uma outra base de dados que tivesse a capacidade de representar o tráfego real de uma rede de computadores contemporânea. Para isso, a base de dados ISCX 2012 (Shiravi et al., 2012) foi utilizada na metodologia desta proposta.

A proposta da base de dados ISCX 2012 foi suprir algumas deficiências encontradas nas bases anteriormente propostas (Ex.: CAIDA, DARPA e KDD). Portanto, essa base de dados oferece os registros das conexões (normais ou intrusivas), todo o tráfego capturado (sem remoção dos payloads), além da captura ser realizada em ambiente real. Além da capacidade de reprodução dos pacotes de rede, a base de dados conta com o tráfego capturado durante 1 semana completa, totalizando 2.450.324 conexões. Durante a captura do tráfego diversos protocolos foram utilizados, tais como: FTP, HTTP, HTTPS, DNS, Netbios, POP3, SMTP, SNMP, SSH e outros.

Para representar os ataques, foram criados 4 cenários reproduzidos separadamente: Ataques que consistem em explorar vulnerabilidades em aplicações (Exploits); Ataques de Negação de Serviço (DoS); Ataques de Negação de Serviço Distribuído (DDoS); e Força Bruta Shiravi et al. (2012). 
Para aplicar a base de dados escolhida utilizando técnicas de Inteligência Computacional, é importante avaliar a distribuição dos dados nas classes (normal ou intrusão). Resultados empíricos mostram um alto grau de desbalanceamento desta base de dados. Sendo assim, utilizou-se o método oversampling que consiste na duplicação dos dados da classe minoritária com objetivo de equilibrar com a quantidade de amostras da classe majoritária.

\subsection{API de Captura e Tratamento de Pacotes e Conexões}

Para que fosse possível a extração de características dos pacotes de rede brutos, assim como são apresentados na base de dados ISCX 2012 (Shiravi et al., 2012), foi necessário o desenvolvimento de uma aplicação que capturasse os pacotes, realizasse o pré-processamento e enviasse para um classificador (SVM). Desta forma, propõe-se uma API Open-Source com o objetivo de possibilitar o préprocessamento de bases de dados existentes, a criação de novas bases de dados e a detecção online de intrusões. A API foi disponibilizada ${ }^{3}$ para a comunidade com o objetivo de permitir que outros autores consigam gerar as suas próprias bases de dados de forma simplificada e, principalmente, com a capacidade de alterar os parâmetros e a extração de características dos pacotes a qualquer momento. Algumas propostas semelhantes foram analisadas ((Salem et al., 2014), (Zhang and Zulkernine, 2005)), porém, a necessidade de alterações constantes e a diferenciação do método motivaram o desenvolvimento de uma API deste tipo.

De acordo com Salem et al. (2014), uma metodologia eficaz para o pré-processamento de uma base de dados de pacotes de rede, com objetivo de detectar ataques, principalmente de negação de serviço, é utilizando vetores de conexão ao invés de analisar apenas pacotes individualmente. Sendo assim, é possível criar uma representação do tráfego de uma janela de tempo (conexão) e analisá-la como um conjunto. Desta forma, a API tem como principal objetivo capturar os pacotes da rede (com a definição de um filtro) e processá-los de forma que sejam formadas diversas "conexões" oriundas de diversos pacotes. O conceito de "conexão" é definido por um fluxo de dados, identificado pela variável Unique_id. Assim, o Unique_id, para os protocolos TCP e UDP, é composto por protocolo, endereço IP de origem, porta de origem, endereço IP de destino e porta de destino. Já no caso do protocolo ICMP, o qual não contém portas em seu cabeçalho, os campos porta de origem e porta de destino foram substituídos pelo ICMP ID (se presente no cabeçalho, caso contrário é preenchido com "-1"). Alguns exemplos da formatação do Unique_id são mostrados a seguir:

- TCP-177.105.60.1:5800-177.60.20.30:80;

- UDP-177.105.60.1:44000-177.60.23.31:6505;

- ICMP-177.60.23.32-177.105.60.1;1200;

- ICMP-177.60.23.32-177.105.60.1;-1.

A seleção de características utilizadas, extraídas da base de dados pela API, para o treinamento da técnica de SVM, foi realizada baseando-se nas propostas de Moustafa and Slay (2016) e Salem et al. (2014). O conjunto de características de conexão, utilizadas nos vetores de conexão, pode ser

\footnotetext{
3 Disponível em: https://github.com/heitorscalco/NIDSProject
}

dividido em 3 categorias: Características obtidas de uma conexão (Tabela 1); Características obtidas por um Buffer de tempo de conexões, em um passado de 2 segundos; (Tabela 2) e Características obtidas por um Buffer das 100 últimas conexões (Tabela 3). O funcionamento da API é apresentado, de forma simplificada, na Figura 5.

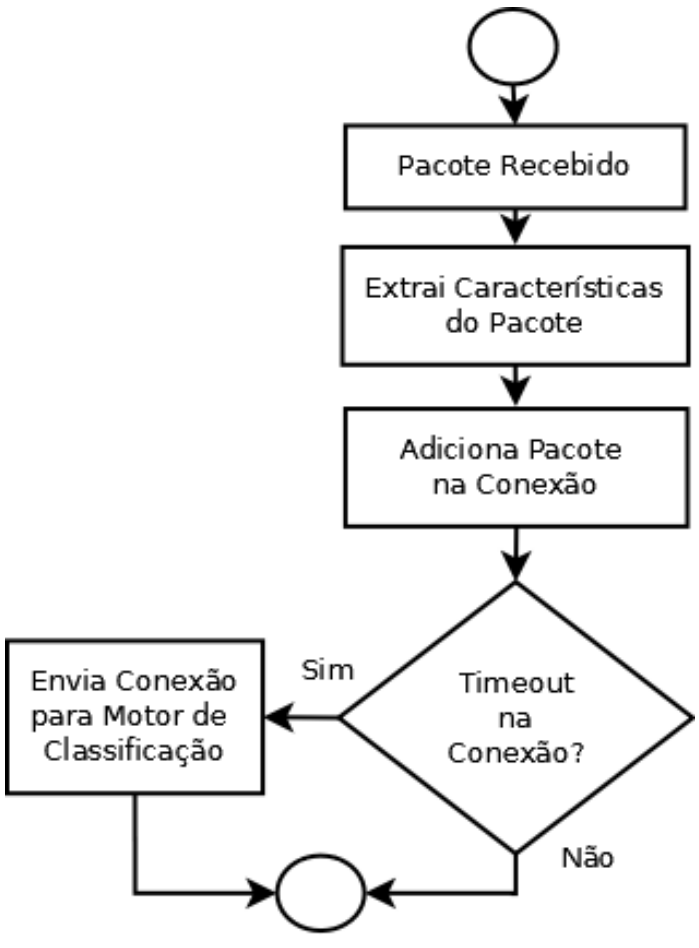

(a) Extração de Características e Pré-processamento

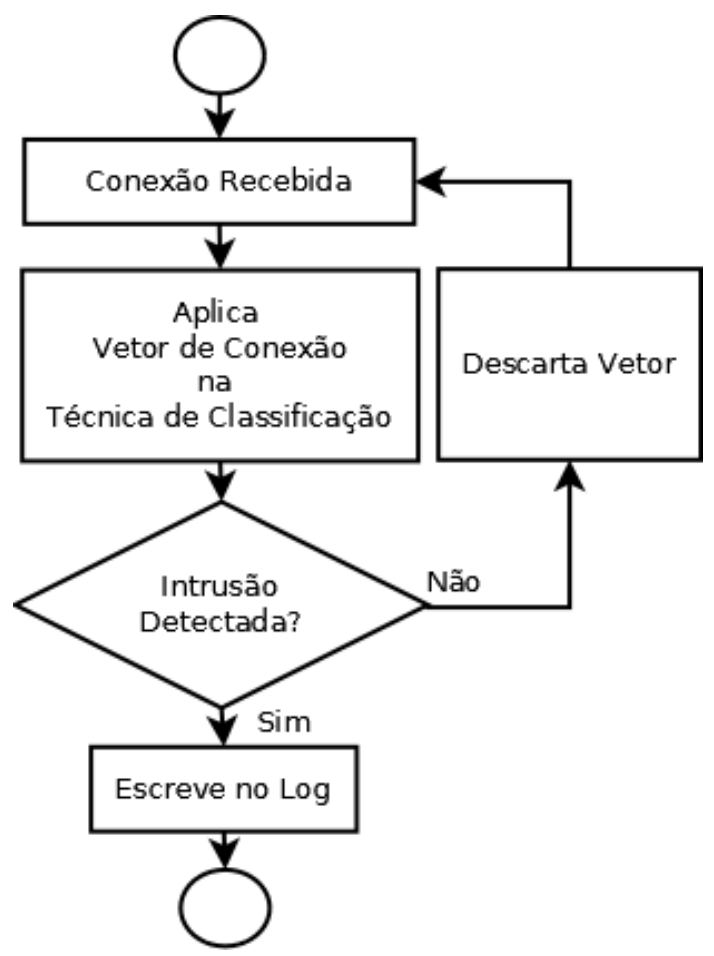

(b) Motor de Classificação e $\log$

Figura 5. Fluxogramas Simplificados da API (a) e (b)

Por fim, o formato dos dados de saída da aplicação, que são disponibilizados para as técnicas de Inteligência 
Computacional, via socket, são apresentados seguindo o modelo dos tópicos abaixo, os quais representam os vetores de conexão:

- 63.0,TCP,HTTP,S0,280,[...],0.0,93.0,100.0,6.0,normal;

- 0.0,TCP,SSH,S0,[...],30100.0,5.0,0.0,100.0,ataque;

Tabela 1. Características da Conexão

\begin{tabular}{llcl}
\hline$\#$ & Característica & Descrição & Tipo \\
\hline 1 & Duração & Tempo (em segundos) da conexão & contínuo \\
2 & Protocolo & Protocolo (Ex.: TCP, UDP, ICMP) & discreto \\
3 & Serviço & Serviço utilizado (determinado pela porta) & discreto \\
4 & Flag da Conexão & Estado da Conexão (Ex.: Handshake) & discreto \\
5 & SourceToDest & Bytes enviados da Origem para o Destino & contínuo \\
6 & DestToSource & Bytes enviados do Destino para a Origem & contínuo \\
7 & Land & 1 se a conexão é de/para o mesmo destino/porta, 0 o inverso & discreto \\
8 & Wrong & Pacotes com erro de checksum & contínuo \\
9 & Urgent & Pacotes TCP com a Flag Urgent & contínuo \\
10 & STTL & TTL do primeiro pacote da Origem & contínuo \\
11 & DTTL & TTL do primeiro pacote do Destino & contínuo \\
12 & SourceToDestPkts & Pacotes enviados da Origem para o Destino & contínuo \\
13 & DestToSourcePkts & Pacotes enviados do Destino para a Origem & contínuo
\end{tabular}

Tabela 2. Características do Buffer de Tempo (Padrão: 2 segundos)

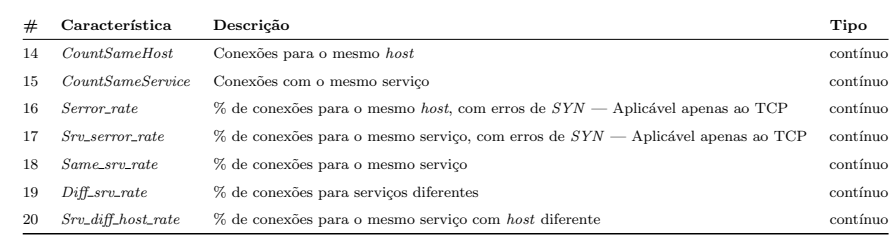

Tabela 3. Características do Buffer de Conexões (Padrão: 100 conexões)

\begin{tabular}{llll}
\hline$\#$ & Característica & Descrição & Típo \\
\hline 21 & Count & Conexōes para o mesmo host & contínuo \\
22 & Srv_count & Conexōes com o mesmo serviço & contínuo \\
23 & Same_srv_rate & \% de conexōes para o mesmo host, com o mesmo serviço & contínuo \\
24 & Diff_srv_rate & \% de conexōes para o mesmo host, com serviços diferentes & contínuo \\
25 & Same_src_port_rate & \% de conexōes com a mesma porta de origem & contínuo \\
26 & Srv_diff_host_rate & \% de conexōes para o mesmo serviço, com host diferente & contínuo \\
27 & Serror_rate & \% de conexōes para o mesmo host, com erro de SYN - Aplicável apenas ao TCP & contínuo \\
28 & Srv_serror_rate & \% de conexōes para o mesmo serviço, com erro de SYN - Aplicável apenas ao TCP & contínuo \\
\hline
\end{tabular}

Os diferentes modos de operação (Online, Captura de Dados e Leitura de Bases de Dados) permitem aos pesquisadores realizar diversos experimentos, sem precisar alterar a API. Cabe ressaltar que, havendo a necessidade de um novo modo de operação, é possível adicionar novos recursos de forma simplificada a API. A próxima seção apresenta o processo de construção de uma nova base de dados de testes utilizando a API desenvolvida.

\subsection{Processo de construção da Base de Dados de Testes}

A base de dados da ISCX 2012 já disponibiliza dados de uma rede de computadores em um ambiente real. Porém, foi necessária a construção de uma nova base de dados com a disposição de infraestrutura (Computadores, Roteadores, Switches, Smartphones) diferente da apresentada pela ISCX 2012. Isto permite realizar experimentos para comprovar que o método é eficaz para diferentes tipos de infraestruturas e dados, não somente pelos disponibilizados nos dados de treinamento.

A infraestrutura de rede, sem dúvida, é um ponto importante para a criação de uma base de dados. É necessário ter cautela para diversos fatores tais como: topologia, serviços, segurança e disponibilidade dos ativos de rede. A infraestrutura utilizada contou com 8 computadores sendo: 1 utilizado para a execução da API; 1 para virtualizar os servidores; e outros 6 para gerar tráfego de rede; além de 2 Smartphones para realizar a utilização de aplicativos. Foi necessário a configuração do espelhamento das portas do switch (Mirroring) para que todo o tráfego pudesse ser capturado (representado pelo "Mirror" da Figura 6). A Figura 6 representa um esboço do ambiente de rede utilizado para a criação da base de dados.

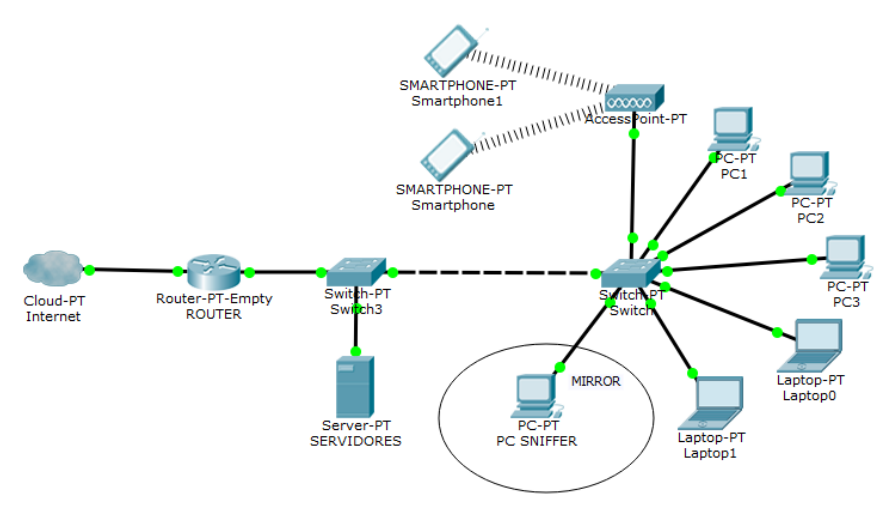

Figura 6. Ambiente de Rede

O Switch, Access Point e o Roteador (Figura 6) foram utilizados sem qualquer configuração de segurança, o que garante a livre transmissão dos pacotes de rede (maliciosos ou não). Os endereços MAC dos computadores e Smartphones foram cadastrados no servidor DHCP para que os endereços IP não sofressem alterações durante o processo de criação da base de dados.

Para que seja possível representar o uso normal e anômalo de uma rede de computadores, é necessário que vários serviços do dia a dia sejam executados durante a construção da base de dados. Desta forma, diversas aplicações foram testadas durante o processo de captura de pacotes, conforme é apresentado na Tabela 4. Também, executou-se ataques de negação de serviço (DoS e DDoS) com os protocolos TCP (utilizando diversas flags), UDP e ICMP, e também varredura de portas. Ao final, obteve-se a captura de 115.030 conexões, quantidade considerada suficiente para realizar testes de eficiência do método proposto.

\subsection{Pré-processamento e Normalização dos dados}

Os processos de normalização e pré-processamento são, sem dúvida, fases muito trabalhosas e importantes para garantir a eficácia do treinamento da técnica de SVM. Como o formato dos dados de saída da API possui algumas características não numéricas, é necessário realizar um simples pré-processamento para transformá-las em entradas numéricas.

Devido a isso, algumas entradas de dados sofrem modificações, como por exemplo, os protocolos (TCP, UDP e ICMP), as Flags de estado das conexões (Handshake, Established, Termination, Closed, [...]) e os nomes dos serviços (HTTP, HTTPS, POP3, SMTP, SNMP, [...]). Como a classificação de vários protocolos, Flags e/ou serviços não são uma grandeza, onde é possível definir 
Tabela 4. Serviços/Protocolos utilizados durante a construção da base de dados

\begin{tabular}{ll}
\hline Ferramenta & Protocolo \\
\hline Navegador Web (Google Chrome e Mozilla Firefox) & HTTP, HTTPS \\
Streaming de Áudio (Spotify) & UDP e TCP \\
Hangouts, Skype e Facebook Call & VOIP (UDP e TCP) \\
Cliente FTP (Filezilla) & FTP \\
Cliente de Email (Outlook, Thunderbird, Gmail (Android)) & POP3, IMAP, SMTP \\
Ferramenta de Monitoramento de Redes (Cacti) & SNMP \\
Cliente SSH (Shell Linux, Putty, WinSCP) & SSH \\
Cliente e Servidor DNS (Bind9 - Linux) & DNS \\
Cliente e Servidor DHCP (ISC DHCP- Linux) & DHCP \\
Sincronização de Horário com Servidor & NTP \\
Descoberta de Rede Microsoft & NETBIOS \\
Cliente MEGA e Dropbox & TCP, HTTP, HTTPS \\
\hline
\end{tabular}

valores de distância entre si, é necessário implementar um algoritmo que adicione uma entrada diferente para cada protocolo. Por exemplo, a entrada do protocolo do dado corrente é preenchido com 1, enquanto as outras entradas de protocolo são preenchidas com o valor 0 . De forma que ( $n$ é o número de Protocolos/Flags/Serviços distintos encontrados nos dados):

- $\mathrm{HTTP}=\left[\begin{array}{llllll}1 & 0 & 0 & 0 & \ldots & n\end{array}\right]$;

- $\operatorname{SMTP}=\left[\begin{array}{llllll}0 & 1 & 0 & 0 & \ldots & n\end{array}\right]$;

- POP3 = $\left[\begin{array}{llllll}0 & 0 & 1 & 0 & \ldots & n\end{array}\right]$

- $\operatorname{HTTPS}=\left[\begin{array}{llllll}0 & 0 & 0 & 1 & \ldots & n\end{array}\right]$.

Dessa forma, os dados de saída da API, que tem 28 variáveis de entrada, passam a ter 96 variáveis de entrada em virtude do grande número de serviços contabilizados, e ainda outras informações que precisam ser partilhadas em entradas (Protocolos e Flags). Por fim, a TAG que apresenta se a conexão é intrusiva ou não, é pré-processada para 0 (Normal) e 1 (Intrusão).

\subsection{Treinamento da Máquinas de Vetores de Suporte}

Em virtude do alto custo computacional da técnica de SVM, foi necessária a utilização de uma solução que proporcione resultados mais rapidamente. Sendo assim, a plataforma Microsoft Machine Learning Studio (Microsoft, 2019) atendeu aos requisitos, a qual conta com uma infraestrutura própria e a implementação de SVM com kernel Linear, entre outros. Os parâmetros de treinamento foram definidos por uma função de busca de parâmetros nativa da plataforma Microsoft Machine Learning Studio (Microsoft, 2019), utilizando cerca de 10\% dos dados de treinamento.

Com intuito de evitar questionamentos a respeito da consistência dos resultados obtidos, os treinamentos foram repetidos por 10 vezes. Cabe ressaltar que, antes de cada treinamento, os dados foram misturados e separados de forma aleatória, mantendo a proporção de dados para treinamento e teste.

\section{RESULTADOS E DISCUSSÃO}

A partir dos experimentos realizados com a metodologia proposta neste artigo, utilizando a base de dados ISCX
2012 (Shiravi et al., 2012) para treinamento e a base de dados de testes criada a partir da API, foi possível obter diversos resultados, os quais são apresentados em detalhes nesta seção.

A utilização da plataforma Microsoft Machine Learning Studio (Microsoft, 2019) demonstrou-se eficaz para o treinamento da técnica de SVM. A duração média dos treinamentos, dada a infraestrutura de alto desempenho do Microsoft Azure (Microsoft, 2019), foi de 12 minutos e 48 segundos.

A Tabela 5 apresenta as taxas de acerto obtidas com as duas bases de dados. É possível perceber a eficiência da técnica de SVM para a detecção de intrusão em redes de computadores utilizando as características propostas neste artigo. Ainda na Tabela 5, percebe-se o pequeno desvio padrão obtido, em relação a todos os treinamentos, afirmando assim a consistência dos resultados. Cabe ressaltar que a base de dados de testes, construída a partir da API, foi utilizada apenas como dados de teste, ou seja, não fizeram parte do treinamento do SVM. Desta forma, é possível perceber a alta capacidade de generalização do método em que, mesmo utilizando dados desconhecidos e de uma infraestrutura diferente, manteve a taxa de acertos elevada.

Tabela 5. Percentual de Acertos utilizando a Base de Dados ISCX 2012 com SVM

\begin{tabular}{cccc}
\hline Treinamento & $\begin{array}{c}\text { Taxa de Acerto } \\
\text { com os } \\
\text { dados de Teste }\end{array}$ & $\begin{array}{c}\text { Taxa de Acerto } \\
\text { com a } \\
\text { Base de dados da API }\end{array}$ & Duração \\
\hline 1 & $95.03 \%$ & $95.90 \%$ & $11 \mathrm{~min}$ e $55 \mathrm{~s}$ \\
2 & $95.06 \%$ & $95.91 \%$ & $13 \mathrm{~min}$ e $05 \mathrm{~s}$ \\
3 & $95.02 \%$ & $95.90 \%$ & $13 \mathrm{~min}$ e $34 \mathrm{~s}$ \\
4 & $95.01 \%$ & $95.90 \%$ & $12 \mathrm{~min}$ e $40 \mathrm{~s}$ \\
5 & $95.04 \%$ & $95.89 \%$ & $12 \mathrm{~min}$ e $01 \mathrm{~s}$ \\
6 & $95.04 \%$ & $95.90 \%$ & $11 \mathrm{~min}$ e $57 \mathrm{~s}$ \\
7 & $95.05 \%$ & $95.90 \%$ & $13 \mathrm{~min}$ e $25 \mathrm{~s}$ \\
8 & $95.09 \%$ & $96.33 \%$ & $13 \mathrm{~min}$ e $48 \mathrm{~s}$ \\
9 & $95.07 \%$ & $95.89 \%$ & $12 \mathrm{~min}$ e $03 \mathrm{~s}$ \\
10 & $95.02 \%$ & $96.05 \%$ & $13 \mathrm{~min}$ e $40 \mathrm{~s}$ \\
Média & $\mathbf{9 5 . 0 4 \%}$ & $\mathbf{9 5 . 9 5 \%}$ & - \\
Desvio Padrão & $\mathbf{0 . 0 2 4 9 7}$ & $\mathbf{0 . 1 3 9 5 3}$ & - \\
\hline
\end{tabular}

Os valores resultantes da busca de parâmetros obtidos por meio da biblioteca nativa da plataforma Microsoft Machine Learning Studio, são apresentados nos itens a seguir:

- Tipo de SVM: C-SVC.

- Kernel: Linear.

- Custo: 0,09.

- Gamma: não se aplica.

- Número de Iterações: 100.

- Porção para realizar o treinamento: $80 \%$.

- Porção para testar o treinamento: $20 \%$.

As Tabelas 6 e 7 apresentam as matrizes de confusão.É possível perceber o número reduzido de falsos positivos e falsos negativos, em ambos os casos (treinamento e teste). Cabe ressaltar que a pior situação, em um NIDS, é a ocorrência de Falsos Negativos. Essa é uma situação em que um ataque está passando pela rede e, mesmo assim, 
o NIDS não é capaz de detectá-lo. Os valores de Falsos Negativos apresentados pelas Tabelas 6 e 7 afirmam a plausibilidade do método proposto.

Tabela 6. Matriz de Confusão do NIDS com a Base de Dados ISCX 2012 (Primeiro Treinamento) com SVM

\begin{tabular}{rccc}
\hline & Positivo & Negativo & Total \\
\hline Positivo & $391389(48.60 \%)-V P$ & $10112(1.25 \%)-F N$ & 401501 \\
Negativo & $29890(3.71 \%)-F P$ & $373773(46.42 \%)-V N$ & 403663 \\
Total & 421279 & 383885 & 805164 \\
\hline
\end{tabular}

Tabela 7. Matriz de Confusão do NIDS com a Base de Dados da API (Primeiro Treinamento) com SVM

\begin{tabular}{rccr}
\hline & Positivo & Negativo & Total \\
\hline Positivo & $62719(54.52 \%)-V P$ & $406(0.35 \%)-F N$ & 63125 \\
Negativo & $4315(3.75 \%)-F P$ & $47590(41.37 \%)-V N$ & 51905 \\
Total & 67034 & 47996 & 115030 \\
\hline
\end{tabular}

Já a Figura 7 apresenta a análise da importância das características da base de dados, em relação ao primeiro treinamento. Os demais valores foram excluídos do gráfico, por não apresentarem importância significativa nos resultados (valores resultantes muito próximos a zero, ou zero). As abreviações "BC" e "BT" significam a origem das características, que representam o Buffer de Conexões (Tabela 3) e Buffer de Tempo (Tabela 2), respectivamente. Essa informação foi obtida utilizando uma função (Permutation Feature Importance), nativa da plataforma Microsoft Machine Learning Studio (Microsoft, 2019), a qual recebe como parâmetro o modelo treinado e a base de dados.

Figura 7. Gráfico da importância das principais características da Base de Dados ISCX 2012, utilizando SVM (Primeiro Treinamento)

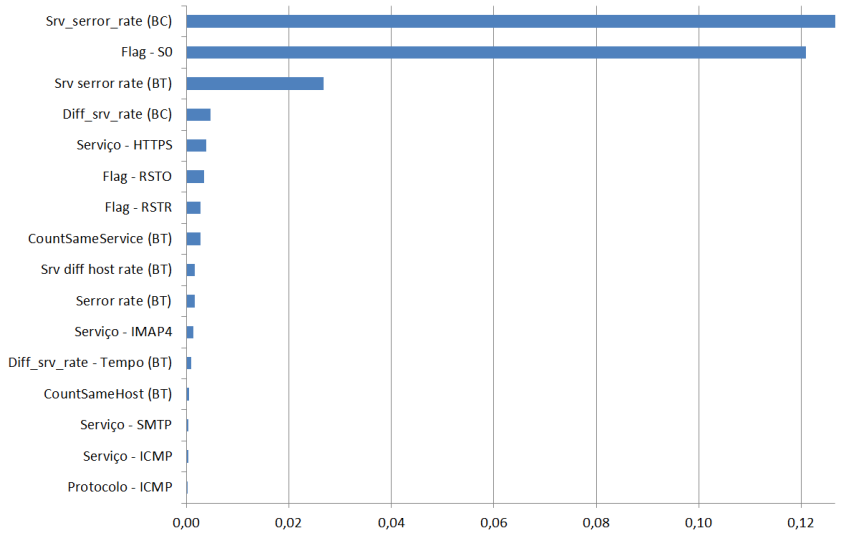

De acordo com Araújo et al. (2013), valores do coeficiente Kappa acima de 0,75 representam um classificador perfeito, em que os resultados apresentam consistência e indicando que não foram obtidos por mero acaso. Os resultados obtidos do coeficiente Kappa foram de 0,90 para a base de dados da ISCX e 0,91 para a base de dados da API, levando em consideração a estrutura do SVM no primeiro treinamento.
A Tabela 8 apresenta alguns resultados com metodologias semelhantes, utilizando a base de dados ISCX e a técnica de SVM. Percebe-se que este artigo tem como principal diferencial a validação dos resultados em uma base de dados alternativa, criada em ambiente real, além da validação com a base de dados ISCX.

Tabela 8. Taxas de Acerto de Trabalhos Correlatos

\begin{tabular}{rcc}
\hline & Base de dados ISCX & Base de dados Alternativa \\
\hline Nossa Proposta & $95.04 \%$ & $95.95 \%$ \\
Ullah and Mahmoud (2017) & $99.64 \%$ & - \\
Heidarian et al. (2015) & $89.25 \%$ & - \\
\hline
\end{tabular}

\section{CONCLUSÃO}

Este artigo teve como principal objetivo explorar, avaliar, apresentar e validar a utilização da técnica de Máquinas de Vetores de Suporte em problemas de detecção de intrusão em redes de computadores. Os resultados obtidos dos valores de taxas de acerto, matrizes de confusão e coeficientes Kappa comprovam a eficiência da utilização de Máquinas de Vetores de Suporte com as características propostas na detecção de intrusão em redes de computadores, tanto no ambiente real quanto simulado.

A API desenvolvida demonstrou ser uma grande aliada ao meio científico pois permite a criação de novas bases de dados de tráfego de rede e também o pré-processamento de bases existentes. Desta forma, abre-se uma oportunidade para outros pesquisadores conseguirem extrair características das conexões de rede e aplicar nos métodos de classificação desejados.

Como propostas de continuidade, recomenda-se realizar experimentos com SVM One-Class, sendo possível a obtenção de resultados ainda mais satisfatórios.

\section{AGRADECIMENTOS}

Os autores agradecem a FAPEMIG (Fundação de Amparo à Pesquisa do Estado de Minas Gerais) e a Universidade Federal de Lavras pelo aporte financeiro para a realização deste trabalho.

\section{REFERÊNCIAS}

Araújo, N.V.S., Shinoda, A.A., Oliveira, R., Ferreira, E.T., and Nascimento, V.E. (2013). Kappa-ARTMAP Fuzzy: uma metodologia para detecção de intrusos com seleção de atributos em redes de computadores. In Anais do XVIII Workshop de Gerência e Operação de Redes e Serviços, p. 119 - 130. Universidade de Brasília, SBRC, Brasilia - DF, Brasil. URL http://sbrc2013.unb.br/ files/anais/wgrs/artigos/artigo-9.pdf. Acesso em abril de 2019.

Ben-Hur, A. and Weston, J. (2010). A User's Guide to Support Vector Machines, 223-239. Humana Press, Totowa, NJ. doi:10.1007/978-1-60327-241-4_13.

CERT.BR (2019). Centro de Estudos, Resposta e Tratamento de Incidentes de Segurança no Brasil. URL http://www. cert.br/. Acesso em abril de 2019.

Heidarian, Z., Movahedinia, N., Moghim, N., and Mahdinia, P. (2015). Intrusion detection based on normal 
traffic specifications. International Journal of Computer Network and Information Security, 7(9), 32.

Hsu, C.W., Chang, C.C., Lin, C.J., et al. (2003). A practical guide to support vector classification. Technical report, Department of Computer Science, National Taiwan University. URL https://www.csie.ntu.edu. tw/ $c j l i n /$ papers/guide/guide.pdf. Acesso em abril 2019.

Karen Scarfone, P.M. (2007). Guide to Intrusion Detection and Prevention Systems (IDPS). National Institute of Standards and Technology Gaithersburg. URL https://ws680.nist.gov/publication/ get_pdf .cfm?pub_id=50951. Acesso em abril de 2019.

Microsoft (2019). Microsoft Azure Machine Learning Studio. URL https://studio. azureml.net. Acesso em abril de 2019.

Moustafa, N. and Slay, J. (2016). The evaluation of Network Anomaly Detection Systems: Statistical analysis of the UNSW-NB15 data set and the comparison with the KDD99 data set. Information Security Journal: A Global Perspective, 1-14.

Rodrigues, R.C.B., Viana, R., Pasquali, A., Pistori, H., and Alvarez, M.A. (2007). Máquinas de vetores de suporte aplicadas à classificação de defeitos em couro bovino. In Anais do III Workshop de Visão Computacional (WVC). São José do Rio Preto, São Paulo. URL http://www.gpec.ucdb.br/pistori/ publicacoes/rodrigues_wvc2007.pdf. Acesso em abril de 2019.

Salem, M., Reissmann, S., and Buehler, U. (2014). Persistent dataset generation using real-time operative framework. In International Conference on Computing, Networking and Communications (ICNC), 1023-1027. IEEE. doi:10.1109/ICCNC.2014.6785478.

Santos, E.M.d. (2002). Teoria e Aplicação de Support Vector Machines à Aprendizagem e Reconhecimento de Objetos Baseado na Aparência. Ph.D. thesis, Universidade Federal da Paraíba.

Scalco Neto, H., Lacerda, W.S., and Boell, V.G. (2015). Reconhecimento de Intrusão em Redes de Computadores utilizando Pybrain. In Anais do 12 Congresso Brasileiro de Inteligência Computacional, 1-6. ABRICOM. doi:10.21528/CBIC2015-051.

Shalev-Shwartz, S., Singer, Y., Srebro, N., and Cotter, A. (2011). Pegasos: Primal estimated sub-gradient solver for SVM. Mathematical programming, 127(1), 3-30.

Shiravi, A., Shiravi, H., Tavallaee, M., and Ghorbani, A.A. (2012). Toward developing a systematic approach to generate benchmark datasets for intrusion detection. Computers \& Security, 31(3), 357-374.

Stolfo, S.J., Fan, W., Lee, W., Prodromidis, A., and Chan, P.K. (2000). Cost-based modeling for fraud and intrusion detection: results from the JAM project. In Proceedings DARPA Information Survivability Conference and Exposition. DISCEX'00, volume 2, 130-144. doi:10.1109/DISCEX.2000.821515. URL http://ids.cs.columbia.edu/sites/default/ files/ada511232.pdf. Acesso em abril de 2019.

Uchoa, J.Q. (2009). Algoritmos Imunoinspirados aplicados em segurança computacional: utilizaçao de algoritmos inspirados no sistema imune para detecçao de intrusos em redes de computadores. Ph.D. thesis, UFMG, Belo Horizonte.
Ullah, I. and Mahmoud, Q.H. (2017). A filter-based feature selection model for anomaly-based intrusion detection systems. In 2017 IEEE International Conference on Big Data (Big Data), 2151-2159. IEEE.

Wang, J. (2009). Computer network security: theory and practice. Springer Publishing Company, Incorporated, New York. 384 p.

Zhang, J. and Zulkernine, M. (2005). Network intrusion detection using random forests. In Proceedings of $3 \mathrm{rd}$ Annual Conference Privacy, Security and Trust (PST), pp. 53-61. St. Andrews, New Brunswick, Canada. 